\title{
Efectos de tres densidades de siembra y disponibilidad de alimento sobre el desarrollo y sobrevivencia de larvas de yaque, Leiarius marmoratus (Pisces: Pimelodidae)
}

\author{
Effects of three planting densities and food availability on the \\ development and survival of larval yaque, Leiarius marmoratus (Pisces: \\ Pimelodidae) \\ Toro Freddy $A^{1}$, Cruz Pablo E. ${ }^{2}$ y Gallego Fernando ${ }^{3}$ \\ 'Zootecnista, estudiante MSc, ${ }^{2} \mathrm{PhD}$ Director Instituto de Investigaciones de la \\ Orinoquia Colombiana (IIOC), ${ }^{3} \mathrm{PhD}$ Docente UDCA \\ ftoro@unillanos.edu.co
}

Recibido 8 de Febrero 2012, Aprobado 18 de Abril 2012

\section{RESUMEN}

Esta investigación se desarrollo en el laboratorio de producción de alimento vivo del instituto de Acuicultura (IALL) de la Universidad de los Llanos (UNILLANOS), localizado en el kilometro 4 vía Puerto López, vereda Barcelona del municipio de Villavicencio (Meta) a 418 m.s.n.m. Consideró como objetivos evaluar el desempeño productivo de larvas de yaque (Leiarius marmoratus) bajo tres densidades de siembra (15, 30 y 45 larvas/litro) y tres regímenes de suministro de alimento vivo (250, 500 y 750 nauplios/larva), en condiciones controladas de laboratorio; y evaluar la ganancia de peso y talla, la tasa de crecimiento específica, el factor de crecimiento relativo y porcentaje de sobrevivencia del yaque durante la etapa larval, bajo tres condiciones de densidades de siembra y tres regímenes de alimentación. Para el estudio se obtuvieron larvas de yaque por medio de reproducción artificial utilizando los protocolos desarrollados en el Instituto de Acuicultura de los Llanos. Las larvas objeto de estudio se tomaron a las 48 horas post-eclosión, con un $90 \%$ de reabsorción del saco vitelino. Se utilizaron 54 peceras de 4 litros de capacidad, las cuales fueron mantenidas con un volumen de 3 litros de agua, aireación constante y recambio diario del $50 \%$. La temperatura promedio se mantuvo en $27{ }^{\circ} \mathrm{C}$. Se empleo un diseño bifactorial completamente al azar. Los factores evaluados fueron densidades de siembra y concentración de alimento a 
suministrar. Los datos obtenidos fueron procesados por medio de estadística descriptiva como promedio y desviación estándar. Para revisar el efecto de los factores densidad de siembra y concentración de comida, se realizo un análisis de varianza (ANOVA) bajo los procedimientos GLM del paquete estadístico SAS, revisando los supuestos de normalidad (test de Kolmogorov-Simirnov) y homogeneidad de varianzas (prueba de Levena), además se utilizo el test de Tukey como prueba posterior para comparación de medias entre los diferentes tratamientos. El criterio de significancia se manejo a un nivel de $p<0,05$. Los parámetros como temperatura, conductividad, sólidos totales y oxigeno disuelto, medidos a lo largo del ensayo no presentaron diferencias significativas entre tratamientos; los valores de potencial hidrogenionico $(\mathrm{pH})$ del agua presentaron diferencias significativas, puesto que disminuyeron a medida que se incrementó la concentración de Artemia salina suministrada. Las densidades de siembra que mantuvieron los porcentajes de sobrevivencia más altos fueron de 30 y 45 larvas. $L^{-1}$, con la disponibilidad de 250 nauplios de Artemia salina suministrada. Los resultados obtenidos en este experimento muestran que esta especie tiene una época marcada de canibalismo entre el día 0 y el día 8 . El requerimiento de alimento vivo es una de las mayores limitantes en esta especie. Las densidades manejadas respondieron directamente al tipo y cantidad de alimento utilizado dando como resultado que las densidades más altas con una disponibilidad de alimento adecuada dan una mayor sobrevivencia, aunque su ganancia de peso y talla es relativamente lenta. Para la cadena comercial de silúridos de la región, estos resultados obtenidos favorecen en el mejoramiento del manejo tecnológico de esta especie.

Palabras clave: Larva de yaque, densidad de siembra, ganancia de peso.

\section{ABSTRACT}

This research was developed in the laboratory of live food production of the Institute of Aquaculture (IALL), University of the Llanos (UNILLANOS), located at $\mathrm{Km} 4$ way to Puerto Lopez, Barcelona sidewalk city of Villavicencio (Meta) to 418 m.a.s.l. Considered objectives are to evaluate the productive performance of yaque (Leiarius marmoratus) larvae under three planting densities (15, 30 
and 45 larvae/liter) and three regimes of live food supply (250, 500 and 750 nauplii/larva), in controlled laboratory conditions, and measure the increase in weight and size, the specific growth rate, relative growth factor and survival rate of yaque during the larval stage under three conditions of plant densities and three feeding regimens. For the study were obtained yaque larvae through artificial reproduction using protocols developed at the Instituto de Acuicultura de los Llanos. Larvae under study were taken at 48 hours post-hatching, with $90 \%$ of yolk sac resorption. 54 tanks were used 4 liters of capacity, which were maintained with a volume of 3 liters of water, constant aeration and daily turnover of $50 \%$. The average temperature was maintained at $27^{\circ} \mathrm{C}$. Use twofactor design was completely random. The factors evaluated were plant densities and concentration of food supply. The data were processed using descriptive statistics as mean and standard deviation. To check the effect of the specific density and food concentration, was made an analysis of variance (ANOVA) under the GLM procedures of SAS, reviewing the assumptions of normality (Kolmogorov-Simirnov) and homogeneity of variances (Levena test) also was used as the test of Tukey post-test to compare means between different treatments. The significance criterion was managed at a level of $\mathrm{p}<0.05$. Parameters such as temperature, conductivity, total solids and dissolved oxygen, measured throughout the test did not differ significantly between treatments hydrogenionic potential values $(\mathrm{pH})$ of water showed significant differences, since decreased as concentration increased Artemia salina provided. Stocking densities maintained by the higher survival rates were 30 and 45 larvas.L-1, with the availability of 250 nauplii of Artemia salina provided. The results obtained in this experiment show that this species has marked an era of cannibalism between day 0 and day 8 . The requirement for live food is a major limitation in this species. The densities handled directly responded to the type and amount of feed used with the result that the highest densities with adequate food availability is a greater survival although their weight and height gain is relatively slow. For the commercial chain of Silurids of the region, these results favor the improvement of technological management of this species. 
Keywords: Yaque larval, planting density, weight gain.

\section{INTRODUCCIÓN}

El yaque (Leiarius marmoratus) es un pez nativo de las cuencas de los ríos Amazonas y Orinoco, del orden de los silúridos, familia Pimelodidae (Petrere et al., 2004). Esta especie está asociada con poblaciones de gran actividad migratoria de grandes cuencas a pequeños tributarios y zonas inundadas como esteros y morichales cuando se da la época estacional de lluvias para dar lugar a actividades reproductivas. Esta especie al igual que muchas de las otras especies de bagres no se reproduce en cautiverio. El yaque cuenta con un gran mercado comercial por su valor nutricional, por la calidad de su carne, por su sabor y por su apreciable tamaño siendo la ciudad de Villavicencio centro de acopio de la cuenca de los llanos orientales, para luego ser distribuido a las diferentes ciudades, principalmente a la capital del país (Petrere et al., 2004). Este mercado es sostenido por la sobreexplotación de ríos y zonas inundadas y en otros medios naturales sin control, lo que está generando un deterioro de las poblaciones y conlleva a reducir su número y capacidad de carga, llevándolos a una extinción local.

Los estudios adelantados en esta especie, sólo han permitido conocer algunos aspectos de la biología básica, así como una aproximación hacia la formulación de un protocolo para la inducción de la reproducción bajo condiciones de cautiverio (Díaz et al., 2009). El Instituto de Acuicultura de los Llanos (IALL) de la Universidad de los Llanos viene adelantando un programa de investigación denominado "Diversificación de la piscicultura nacional a través de la introducción de silúridos nativos a los sistemas de producción" trabajando principalmente con yaque (Leiarius marmoratus) y rayado (Pseudoplatystoma fasciatum). Estas investigaciones han sido orientadas a la reproducción en cautiverio, larvicultura, alevinaje, levante y engorde, encontrándose que uno de los mayores desafíos es la producción suficiente y oportuna de alevinos, en donde una larvicultura correcta es la clave de su eficiencia. El éxito de este proceso depende en alto grado de la calidad del agua, de la densidad de 
siembra, disponibilidad de alimento y de la técnica de cultivo (Lopes et al., 2001; Segura et al., 2004).

El yaque (Leiarius marmoratus) en etapa de larvicultura, presenta elevadas tasas de mortalidad (hasta 60\%) debido principalmente a depredación intraespecífica (Díaz et al., 2009), lo anterior conlleva a la heterogeneidad de las tallas, característica inconveniente en este proceso, sin embargo en otras especies de silúridos de hábitos piscívoros, como Pimelodus maculatus (Kennedy y Zaniboni, 2002), Clarias gariepinus y Mystus nemurus (Haylor, 1991; Khan, 1994), se ha logrado superar este inconveniente manipulando la dosis de alimento, aumentando la frecuencia de alimentación, utilizando apropiadas densidades de siembra, realizando selección periódica por talla y proporcionando condiciones ambientales apropiadas (Kestemont et al., 2003; Kennedy y Zanniboni, 2002).

La mayoría de las especies de peces pueden presentar diferentes comportamientos productivos por efecto de la manipulación de la densidad de siembra, de la cantidad y tipo de alimento vivo ofrecido, aún no se conoce con claridad la posible interacción entre estas dos variables y sus efectos en la sobrevivencia y el desarrollo corporal de Leiarius marmoratus. La explotación en general de los silúridos en cautiverio se ha dificultado por su exigencia a las condiciones del agua, su tipo de alimentación y su densidad en el momento de la siembra (Segura et al., 2004), siendo el tipo de alimento el condicionante específico para el cultivo en cautiverio del yaque ( $L$. marmoratus) debido a su preferencia piscívora. El factor más importante que determina el éxito de una explotación, es el manejo de las densidades, ya que se ha demostrado requerimientos de grandes cantidades de espacio siendo especifico para cada especie de pez durante su crecimiento (Khan, 1994). El control de este factor nos permite tener tallas altas en los animales, buenas conversiones alimenticias y sobrevivencias de los silúridos en general.

El pez de cultivo requiere en su dieta proteínas, lípidos, energía, vitaminas y minerales, para el crecimiento, la reproducción y otras funciones fisiológicas normales. Los requerimientos varían entre las especies y dentro de las 
especies en relación a la etapa del ciclo de vida, al sexo a su estado reproductivo y al ambiente. Los requerimientos óptimos de proteína en silúridos en forma general han sido estimados en un rango de $25-52 \%$ dependiendo de la temperatura del agua, disponibilidad de alimento, densidad de siembra, ración de comida, cantidad de energía no proteica y finalmente de la calidad de la proteína en la dieta, así el manejo de niveles de proteína óptimos en las dietas mejora la producción especialmente en especies de hábitos carnívoros (Kim y Lee, 2005).

\section{MATERIALES Y MÉTODOS}

La fase experimental se desarrollo en el laboratorio de producción de alimento vivo del Instituto de Acuicultura (IALL) de la Universidad de los Llanos (UNILLANOS), localizado en el kilometro 4 vía Puerto López, vereda Barcelona del municipio de Villavicencio (Meta) a 418 m.s.n.m. Las condiciones climáticas de la región son características de clima húmedo tropical, con temperatura promedio de $25^{\circ} \mathrm{C}$, precipitación de $4.050 \mathrm{~mm}$ anuales y humedad relativa de $75 \%$. El experimento se realizo durante un periodo de 15 días, comprendiendo la etapa larval de ésta especie. Para el estudio se obtuvieron larvas de yaque (Leiarius marmoratus) (Fotografía 1) por medio de reproducción artificial inducida con extracto de hipófisis de carpa (EHC) utilizando los protocolos desarrollados en el Instituto de Acuicultura de los Llanos. Las larvas objeto de estudio se tomaron a las 48 horas post-eclosión (HPE), con un $90 \%$ de reabsorción del saco vitelino. En el ensayo se utilizaron 54 peceras de 4 litros de capacidad, las cuales fueron mantenidas con un volumen de 3 litros de agua, aireación constante y recambio diario del $50 \%$. La temperatura promedio se mantuvo en $27^{\circ} \mathrm{C}$. Las peceras se recubrían con láminas de plástico de polietileno con el fin de reducir la intensidad de luz y la proliferación de fitoplancton.

En la distribución de los tratamientos, se empleó un diseño bifactorial completamente al azar. Los factores evaluados fueron densidades de siembra y concentración de alimento a suministrar. Las densidades de siembra correspondieron a 15,30 y 45 larvas/L y las concentraciones de alimento vivo 
suministrado fueron de 250, 500 y 750 nauplios/larva, para un total de 9 tratamientos, replicados 6 veces (Tabla 1).

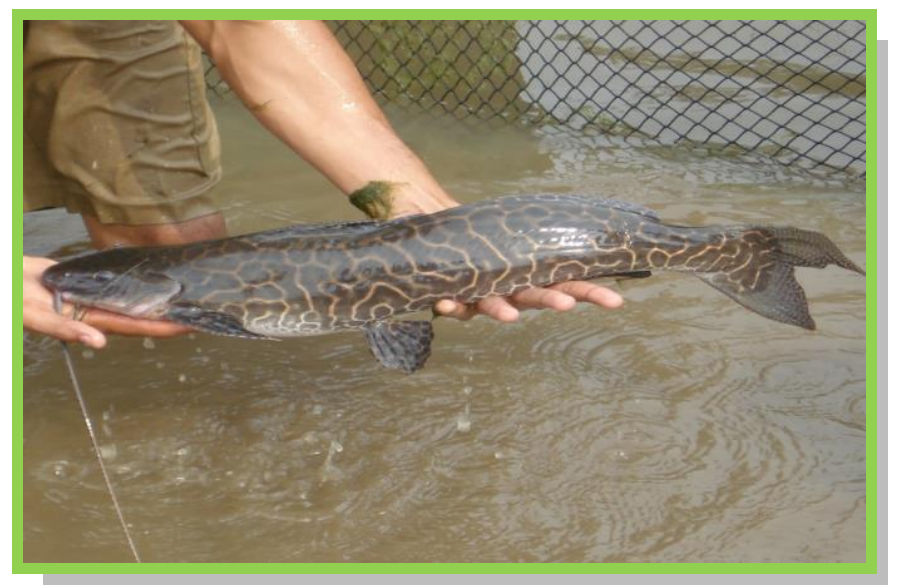

Fotografía 1. Yaque (L. marmoratus). Tomada en la estación.

Tabla 1. Tratamientos de densidades de siembra y concentración de alimento

\begin{tabular}{ccc}
\hline Tratamiento & $\begin{array}{c}\text { Densidad de siembra } \\
\text { (larvas/L) }\end{array}$ & $\begin{array}{c}\text { Concentración de alimento } \\
\text { (nauplios/larva) }\end{array}$ \\
\hline 1 & 15 & 250 \\
2 & 15 & 500 \\
3 & 15 & 750 \\
4 & 30 & 250 \\
5 & 30 & 500 \\
6 & 30 & 750 \\
7 & 45 & 250 \\
8 & 45 & 500 \\
9 & 45 & 750 \\
\hline
\end{tabular}

Como alimento vivo se empleo nauplios de Artemia sp recién eclosionados, los cuales fueron suministrados tres veces al día (7:00, 19:00 y 22:00 horas). Para la eclosión y conteo de los quistes fue utilizada la metodología de Jomori (1999). Lo nauplios una vez eclosionados, después de 24 horas de incubación, fueron suspendidos en 10 litros de agua salinizada, para luego extraer una muestra de $1 \mathrm{ml}$, con la que se hizo una dilución 1:10, en donde se tomaron submuestras de $1 \mathrm{ml}$ para cuantificar el número de nauplios por medio de un estereoscopio, y luego extrapolarlos al volumen inicial obtenido y con ello se hizo la dosificación en los diferentes tratamientos. Cada unidad experimental 
fue sifoneada una hora después del suministro del alimento con el objeto de retirar los desechos y las larvas muertas y así evitar el deterioro del agua.

En todas las unidades experimentales se tomaron diariamente parámetros de calidad de agua como temperatura $\left({ }^{\circ} \mathrm{C}\right)$, potencial hidrogenionico $(\mathrm{pH})$ conductividad $\left(\mu \mathrm{s} . \mathrm{cm}^{-1}\right)$, sólidos disueltos $\left(\mathrm{gr}^{\mathrm{L}} \mathrm{L}^{-1}\right)$, salinidad (ppt) y oxígeno disuelto (mg. $\mathrm{L}^{-1}$ ) por medio de una sonda multiparamétrica (YSI) la sobrevivencia fue estimada por medio de conteos al día, 8 y 15 teniendo en cuenta animales muertos obtenidos diariamente en las labores de mantenimiento de las unidades experimentales. Tanto al inicio como al final del ensayo se realizó aleatoriamente fijación de larvas en formol buferado tamponado al $4 \%$ para posterior biometría (Longitud total $(\mathrm{mm})$ y peso corporal (mg) por medio de un ocular de estereoscopio graduado y una balanza analítica (Ohaus, precisión de 0,1 mg) con el fin de obtener las variables productivas.

Las variables planteadas fueron determinadas por medio de las fórmulas de Kesmont y Stalmans, (1992): \% sobrevivencia: (Nro final de larvas x 100) / Nro inicial de larvas; Ganancia de peso (GP): Peso final (mg) - Peso inicial (mg); Ganancia diaria de peso (GDP mg día $a^{-1}$ ): (Peso final - Peso inicial) / días del ciclo productivo; Tasa de crecimiento específico (TCE): $100 \times$ (In Wtf - In Wti) / $\Delta t$, donde, Wtf: Peso final, Wti: Peso inicial, $\Delta t$ : Duración en días entre una biometría y otra, In: Logaritmo natural; Factor de crecimiento relativo (FCR): Peso final (mg) / longitud total final (cm).

Los datos obtenidos fueron procesados por medio de estadística descriptiva como promedio y desviación estándar. Para revisar el efecto de los factores densidad de siembra y concentración de comida, se realizó un análisis de varianza (ANOVA) bajo los procedimientos GLM del paquete ESTADISTICO SAS, revisando los supuestos de normalidad (test de Kolmogorov-Simirnov) y homogeneidad de varianzas (prueba de Levena), además se utilizo el test de Tukey como prueba posterior para comparación de medias entre los diferentes tratamientos. El criterio de significancia se manejo a un nivel de $p<0,05$. 


\section{RESULTADOS Y DISCUSIÓN}

Los parámetros como temperatura, conductividad, sólidos totales y oxigeno disuelto (Tabla 2), medidos a lo largo del ensayo no presentaron diferencias significativas entre tratamientos; los valores de potencial hidrogenionico $(\mathrm{pH})$ del agua presentaron diferencias significativas, puesto que disminuyeron a medida que se incrementó la concentración de Artemia salina suministrada.

En un ensayo realizado en $P$. fasciatum la respuesta de las variables productivas varía de acuerdo con las densidades de siembra y la cantidad de alimento disponible (Díaz et al., 2009), lo cual también coinciden en los porcentajes de sobrevivencia dados en el trabajo con L. marmoratum, sin embargo, la falta de alimento podría generar canibalismo. Igualmente se puede inferir que la cantidad de alimento no necesariamente está relacionada con la densidad de siembra, ya que factores como la calidad del agua pueden incidir en el comportamiento productivo de la especie.

La Figura 1 ilustra el porcentaje acumulado de sobrevivencia durante los 15 días del ensayo, para las tres densidades de siembra y las tres disponibilidades de Artemia. Los valores de sobrevivencia finales entre los tratamientos presentaron diferencias significativas tanto para la densidad de individuos como para la disponibilidad de alimento $(P<0,001)$. Como se observa en la Figura $1 \mathrm{a}$, estos valores disminuyeron en el octavo día del ensayo de 15 larvas. $L^{-1}$, llegando a valores inferiores a $22 \%$ en las concentraciones 500 y 750 nauplios de Artemia en contraste con las de menor concentración 250 nauplios, la sobrevivencia en ese momento fue alrededor de $40 \%$. Por su parte, los mayores porcentajes de sobrevivencia se observaron en aquellos tratamientos en los cuales no se aumentó la disponibilidad de alimento y se utilizaron las mayores densidades de individuos por litro, es decir, disponibilidad de 250

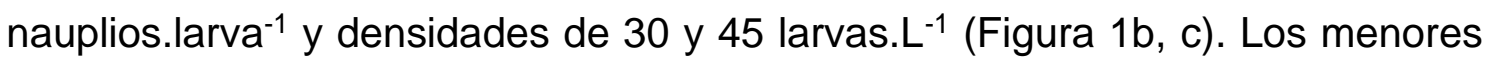
porcentajes de sobrevivencia fueron observados en los tratamientos que

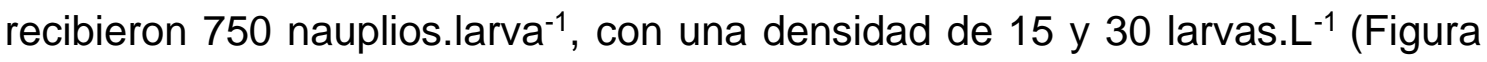
$1 \mathrm{a}, \mathrm{b})$. Las densidades de siembra que mantuvieron los porcentajes más altos 
fueron de 30 y 45 larvas. $\mathrm{L}^{-1}$, con la disponibilidad de 250 nauplios de Artemia salina suministrada (Figura 1b, c).

Tabla 2. Parámetros físico-químicos del agua, para cada parámetro, los valores corresponden a la media \pm SD

\begin{tabular}{ccccccc}
\hline Larvas & $\begin{array}{c}\text { Nauplios } \\
\text { / Larva }\end{array}$ & $\begin{array}{c}\text { Temperatura } \\
\left({ }^{\circ} \mathbf{C}\right)\end{array}$ & $\begin{array}{c}\text { Conductividad } \\
(\boldsymbol{\mu s} . \mathbf{c m})\end{array}$ & TDS & $\begin{array}{c}\text { Oxígeno } \\
\text { disuelto } \\
(\mathbf{m g ~ L})\end{array}$ & pH \\
\hline \multirow{3}{*}{15} & 250 & $26,49 \pm 0,76$ & $199,73 \pm 96,31$ & $0,12 \pm 0,06$ & $3,01 \pm 1,35$ & $7,2 \pm 0,37^{\mathrm{a}}$ \\
& 500 & $26,37 \pm 0,46$ & $203,80 \pm 96,58$ & $0,16 \pm 0,14$ & $3,18 \pm 1,48$ & $7,48 \pm 0,23 \mathrm{~b}$ \\
& 750 & $26,26 \pm 0,57$ & $202,26 \pm 92,13$ & $0,15 \pm 0,11$ & $3,12 \pm 1,25$ & $7,18 \pm 0,14 \mathrm{bc}$ \\
& 250 & $26,12 \pm 0,65$ & $201,46 \pm 92,19$ & $0,12 \pm 0,05$ & $3,32 \pm 1,32$ & $7,15 \pm 0,13^{\mathrm{bc}}$ \\
& 50 & $26,32 \pm 0,44$ & $204,86 \pm 88,86$ & $0,17 \pm 0,17$ & $3,15 \pm 1,15$ & $7,15 \pm 0,18^{\mathrm{bc}}$ \\
& 750 & $26,23 \pm 0,43$ & $209,53 \pm 90,01$ & $0,17 \pm 0,16$ & $3,06 \pm 1,18$ & $6,98 \pm 0,15^{\mathrm{cd}}$ \\
& 250 & $26,02 \pm 0,55$ & $192,73 \pm 80,60$ & $0,12 \pm 0,05$ & $3,23 \pm 1,06$ & $7,15 \pm 0,20^{\mathrm{bc}}$ \\
& 55 & $26,11 \pm 0,72$ & $193,86 \pm 85,63$ & $0,12 \pm 0,05$ & $2,90 \pm 1,19$ & $6,99 \pm 0,16^{\mathrm{cd}}$ \\
& 750 & $26,43 \pm 0,60$ & $211,00 \pm 96,23$ & $0,17 \pm 0,18$ & $2,80 \pm 0,74$ & $6,87 \pm 0,16^{\mathrm{d}}$ \\
\hline
\end{tabular}

Superíndices con letras diferentes entre tratamientos indican diferencias significativas, $(p<0.001)$

Las diferencias morfológicas que contribuyen a la heterogeneidad en el tamaño, son atribuidas a desoves donde la eclosión demora un largo periodo de tiempo, dando origen a un crecimiento asincrónico que permite que las primeras larvas desarrollen rápidamente su boca y por tanto la relación bocatamaño del cuerpo les proporciona mayores habilidades competitivas al momento de consumir larvas ligeramente más pequeñas (Canibalismo tipo I) (Baras y Jobling, 2002; Kestemont et al., 2003) siendo más evidente en el octavo día según los resultados de este trabajo, sumado al desarrollo temprano de las larvas, mejores habilidades de nado y escape (Baras y Jobling, 2002).

Según Núñez et al., (2008), uno de los factores que afecta la sobrevivencia en larvas de $P$. fasciatum, es la regulación del fotoperiodo (aumentando los periodos de oscuridad) junto con las frecuencias alimenticias (aumentando el número de raciones) y no la densidad de siembra. De esta forma, condiciones de oscuridad reducirían significativamente la conducta agresiva, puesto que 
permanecen por largos periodos de tiempo en reposo luego de cada comida (Núñez et al., 2008).
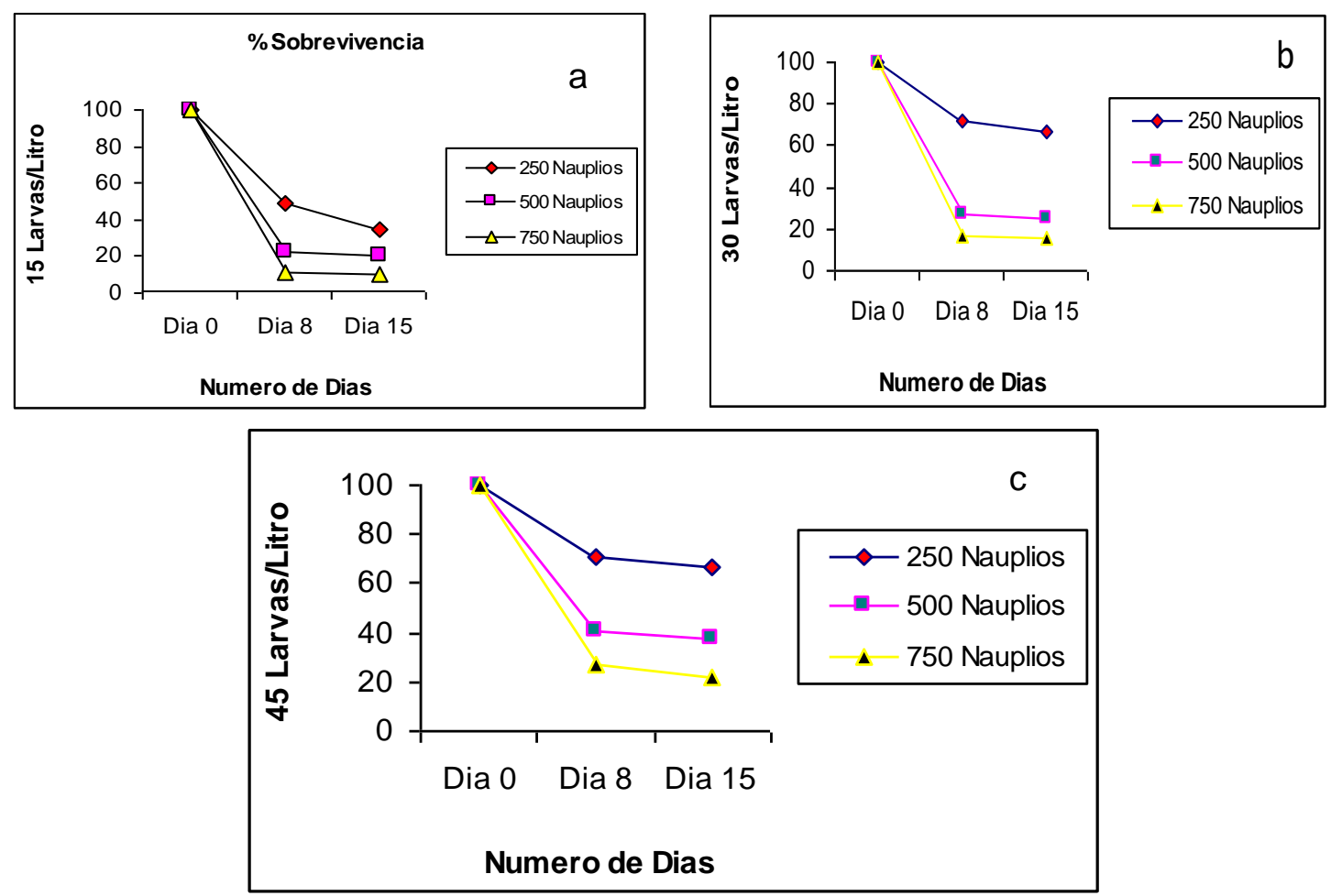

Figura 1. Porcentajes acumulados de sobrevivencia de larvas de yaque (Leiarius marmoratus) alimentadas con diferentes concentraciones de nauplios de Artemia salina

Katavic et al., (1989) evaluaron el efecto de frecuencia de la alimentación en Seabass en la tasa de canibalismo, concluyeron que el canibalismo fue significantemente menor cuando el alimento era ofrecido entre tres y seis veces por día. La observación del experimento realizado, permite concluir que el aumento en la frecuencia de alimentación, mantiene a las larvas satisfechas por el tiempo entre comidas, disminuyendo el canibalismo.

Investigaciones recientes en larvas de $P$. fasciatum alimentadas con nauplios de Artemia salina muestran sobrevivencias del $91 \%$ a los 15 días posteclosión (DPE), y del 65\% con zooplancton natural; sin embargo, a los 28 DPE, esta sobrevivencia disminuye al $50 \%$ en los tratamientos que recibieron nauplios de Artemia, y al $2 \%$ en los tratamientos que recibieron zooplancton natural (Núñez et al., 2008). En este trabajo realizado en larvas de L. marmoratun, el mejor desempeño productivo fue observado en los tratamientos que recibieron baja 
cantidad de Artemia salina (250 nauplios.larva ${ }^{-1}$ ) y bajo condiciones de completa oscuridad con densidades medias y altas (30 y 45 larvas. $\mathrm{L}^{-1}$ ) mostrando porcentajes de sobrevivencia de $66 \%$ y $67 \%$ al final del ensayo.

En catfish (Clarias gariepinus) se observó que cuando la disponibilidad del alimento disminuye, la conducta inicial del pez para garantizar los limitados recursos fue de territorialismo, hasta llegar a un punto máximo después del cual las energías fueron orientadas hacia una conducta más efectiva: el canibalismo (Hecht y Appelbaum, 1988). En esta nueva experiencia se observo cuando había baja densidad de animales sin importar la disponibilidad de alimento, esta conducta de canibalismo se presentaba en altos porcentajes.

En la Tabla 3 se presentan los promedios de ganancia de peso, ganancia de talla y ganancia diaria de peso, así como la tasa de crecimiento específico y el factor de crecimiento relativo. Entre las densidades de siembra evaluadas (15, 30 y 45 larvas. $\mathrm{L}^{-1}$ ) se encontraron diferencias significativas para la ganancia de peso $(\mathrm{P}<0,001)$, siendo la densidad de 15 larvas. $L^{-1}$ la que presento mayores valores (Figura 2). Igualmente, se observaron diferencias significativas en la ganancia de peso, por efecto de la disponibilidad de alimento ofrecido (250, 500 y 750 nauplios.larvas ${ }^{-1}$ ), obteniéndose mayor ganancia de peso en tratamientos que recibieron 500 y 750 nauplios. Larva ${ }^{-1}$.

Trabajos realizados con $P$. fasciatum sugieren que altas densidades de siembra afectan negativamente y de forma lineal la ganancia de peso y la mortalidad en mayor proporción por depredación intraespecífica (Segura et al., 2004); sin embargo, según los resultados obtenidos en el experimento, al aumentar la densidad, acompañado de suministro moderado de alimento, buena calidad de agua y baja intensidad de luz, es posible que la sobrevivencia no se vea afectada, aunque la ganancia de peso y la talla puedan verse afectadas por la baja disponibilidad de alimento, generando una descompensación en el crecimiento que puede aumentar el riesgo de canibalismo (Baras y Jobling, 2002).

Las variables densidad de siembra y disponibilidad de alimento también fue significativa $(\mathrm{P}<0.001)$ para esta variable productiva. Igualmente, la ganancia 
de talla (Figura 3) mostró diferencias significativas entre las densidades de siembra $(P<0,001)$, disponibilidades de Artemia $(P<0.001)$ y su respectiva interacción $(P<0.001)$.

Tabla 3. Valores promedio \pm SD de las variables productivas medidas en larvas de Yaque ( $L$. marmoratus) para cada densidad de siembra y concentración de Artemia suministrada.

\begin{tabular}{ccccc}
\hline Larvas.L $^{-1}$ & Nauplios.Larva $^{-1}$ & $\begin{array}{c}\text { Sobrevivencia } \\
(\%)\end{array}$ & $\begin{array}{c}\text { Ganancia en } \\
\text { Peso }(\mathbf{m g})\end{array}$ & $\begin{array}{c}\text { Ganancia en } \\
\text { talla }(\mathbf{m m})\end{array}$ \\
\hline \multirow{3}{*}{15} & 250 & $34^{\mathrm{b}}$ & $76,94 \pm 33,74^{\mathrm{d}}$ & $18,36 \pm 2,62^{\mathrm{d}}$ \\
& 500 & $21^{\mathrm{bc}}$ & $124,32 \pm 41,79^{\mathrm{c}}$ & $22,03 \pm 2,73^{\mathrm{bc}}$ \\
& 750 & $10^{\mathrm{c}}$ & $199,81 \pm 58,22^{\mathrm{a}}$ & $24,86 \pm 2,79^{\mathrm{a}}$ \\
30 & 250 & $67^{\mathrm{a}}$ & $72,54 \pm 22,56^{\mathrm{d}}$ & $18,53 \pm 1,99^{\mathrm{d}}$ \\
& 500 & $25^{\mathrm{bc}}$ & $114,71 \pm 39,29^{\mathrm{c}}$ & $20,69 \pm 2,60^{\mathrm{cd}}$ \\
& 750 & $15^{\mathrm{c}}$ & $172,67 \pm 64,81^{\mathrm{ab}}$ & $23,66 \pm 3,87^{\mathrm{ab}}$ \\
45 & 250 & $66^{\mathrm{a}}$ & $59,44 \pm 38,68^{\mathrm{d}}$ & $15,08 \pm 7,10^{\mathrm{e}}$ \\
& 500 & $38^{\mathrm{b}}$ & $125,41 \pm 43,96^{\mathrm{c}}$ & $21,33 \pm 2,84^{\mathrm{bc}}$ \\
& 750 & $21^{\mathrm{bc}}$ & $164,32 \pm 68,62^{\mathrm{b}}$ & $22,30 \pm 3,58 \mathrm{abc}$ \\
\hline $\begin{array}{l}\text { Superíndices } \\
(\mathrm{p}<0.001)\end{array}$ & con & letras diferentes & entre tratamientos indican diferencias significativas,
\end{tabular}

La mayor ganancia de peso y talla (Figura 2 y 3) se observó en el tratamiento con densidad de siembra de 15 larvas.L L $^{-1}$ y con disponibilidades de Artemia de 500 y 750 nauplios.Larva- ${ }^{-1}$, respectivamente; contrario a lo observado con las densidades de siembra de 30 y 45 larvas.L- ${ }^{-1}$, en donde las ganancias de peso y de talla fueron bajas pero están relacionadas directamente con las altas tasas de sobrevivencia. El trabajo sobre primer alevinaje en Rhamdia sapo muestra que los mejores porcentajes de sobrevivencia y tasas de crecimiento aumentarían con la disminución de la densidad de siembra (Luchini y Salas., 1985).

Los tratamientos 15,30 y 45 larvas. L $^{-1}$ con 750 nauplios. Larva- ${ }^{-1}$ terminaron con altos valores en ganancia de peso y talla pero con bajos porcentajes de sobrevivencia, producto de la depredación intraespecífica. Así mismo la tasa crecimiento específico fue más baja en los tratamientos que presentaron alta sobrevivencia y por tanto disminuyó el factor de crecimiento relativo. 


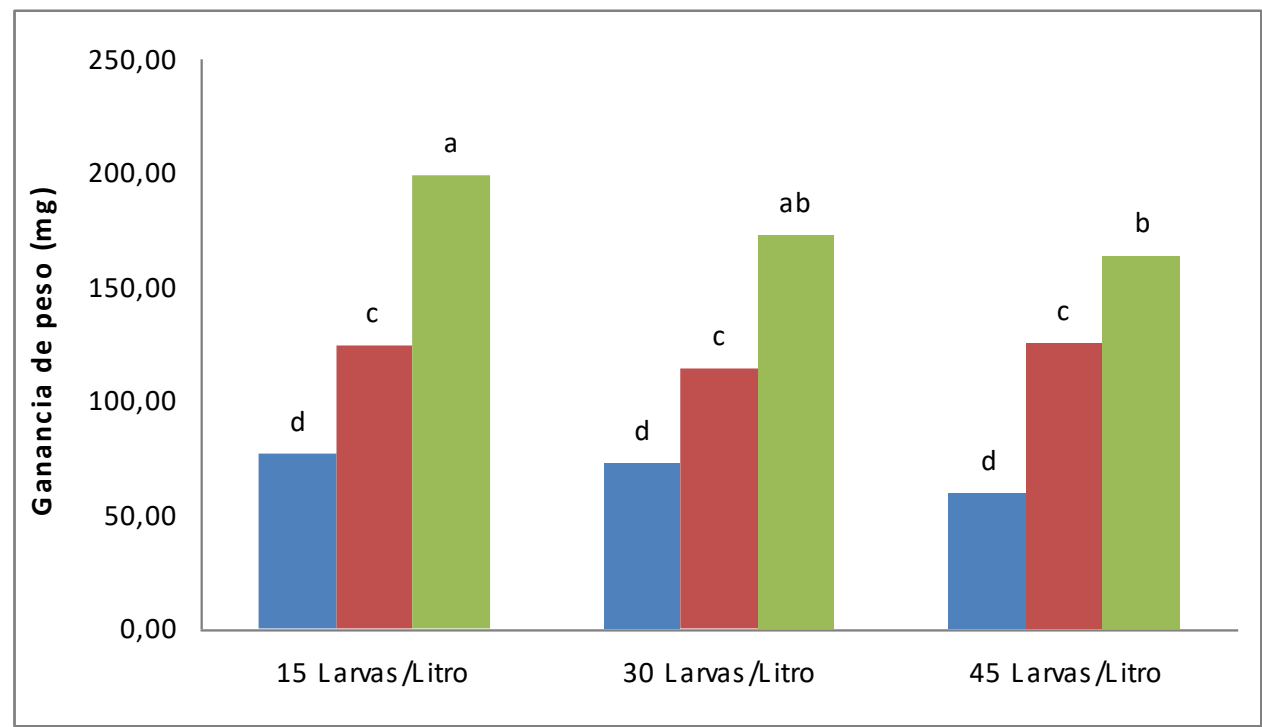

Superíndices con letras diferentes entre tratamientos indican diferencias significativas

Figura 2. Promedio de la ganancia de peso de larvas de yaque ( $L$. marmoratus)

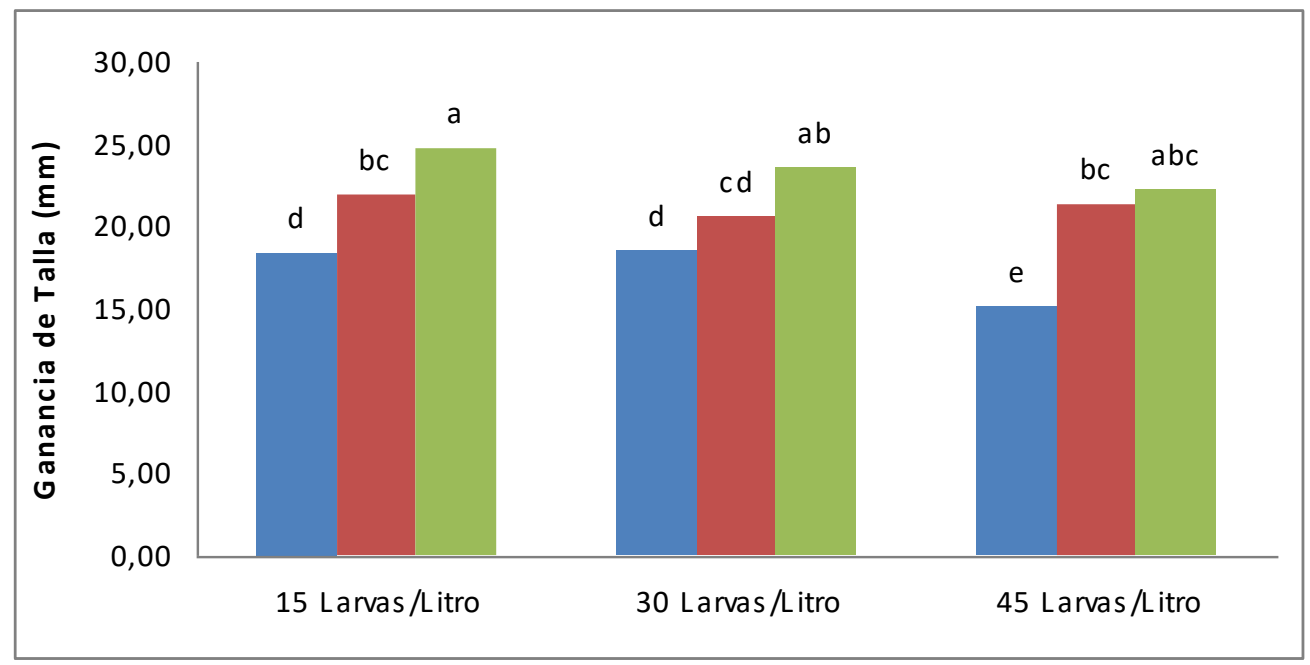

Superíndices con letras diferentes entre tratamientos indican diferencias significativas

Figura 3. Promedio de la ganancia de talla total de larvas de yaque (Leiarius marmoratus)

En el ensayo realizado por Boiani et al., (2003) en Rhamdia quelen a los 15 días posteclosion, la supervivencia de las larvas sembradas en las peceras fue de $76,4 \%$, duplicándose su talla, con una tasa de crecimiento de $205,3 \%$ de la longitud total, estos resultados concluyeron que los mejores resultados de crecimiento frente al control fueron obtenidos en las peceras de menores densidades, resultando en una mayor disponibilidad de espacio y alimento. Con 
la experiencia obtenida en el trabajo realizado, los resultados obtenidos muestran que la tasa de crecimiento está relacionada con la densidad de animales y la cantidad de alimento, sin embargo, la sobrevivencia podría estar relacionada directamente con la cantidad de alimento.

Gallo y García, (2007) en Oreochromis niloticus y Oreochromis mossambicus comentan que el manejo exitoso de altas densidades de cultivo no solo depende de la tolerancia de la especie, y la aplicación de nuevas tecnologías y equipos de producción sino también de una buena nutrición (Alceste et al., 2004) y adecuadas estrategias de alimentación. En el ensayo realizado se pudo observar que: utilizar unas mejores estrategias alimenticias que conlleven a mejorar la ganancia de peso y talla en los primeros 8 días de vida pueden reducir el canibalismo. Esto se podría tener en cuenta para futuros experimentos.

Las Figuras 4 y 5 muestran los factores densidad de siembra y concentración de Artemia, para las variables ganancia de peso y talla respectivamente, observándose que los mejores rendimientos se dieron con el tratamiento de 15 larvas. $\mathrm{L}^{-1}$ alimentadas con 750 nauplios.larva ${ }^{-1}$; sin embargo, al aumentar las densidades de larvas. $\mathrm{L}^{-1}$ la ganancia de peso y de talla disminuyó. En la densidad de 45 larvas. $\mathrm{L}^{-1}$ el tratamiento que recibió 250 nauplios.larva ${ }^{-1}$ presentó los valores más bajos de ganancia de peso.

En el ensayo de Oreochromis niloticus y Oreochromis mossambicus se demostró que el suministro de dietas suplementadas con quistes descapsulados de Artemia, la ración y la frecuencia alimenticia influyen mucho ya que ayudan a mantener saciadas las larvas de Tilapia de ambas especies, reduciendo así, el canibalismo y a la vez aprovechan mejor el alimento reflejando un mejor crecimiento y una menor dispersión en la talla final (Gallo y García, 2007). En el presente trabajo experimental se observó que se mejoraría el crecimiento de los animales, adecuando una mejor frecuencia alimenticia, permitiendo así reducir la mortalidad y disminuir la dispersión de la talla antes del día 8, ya que éste muestra los índices más altos de mortalidad por canibalismo debido a la dispersión de la talla. 


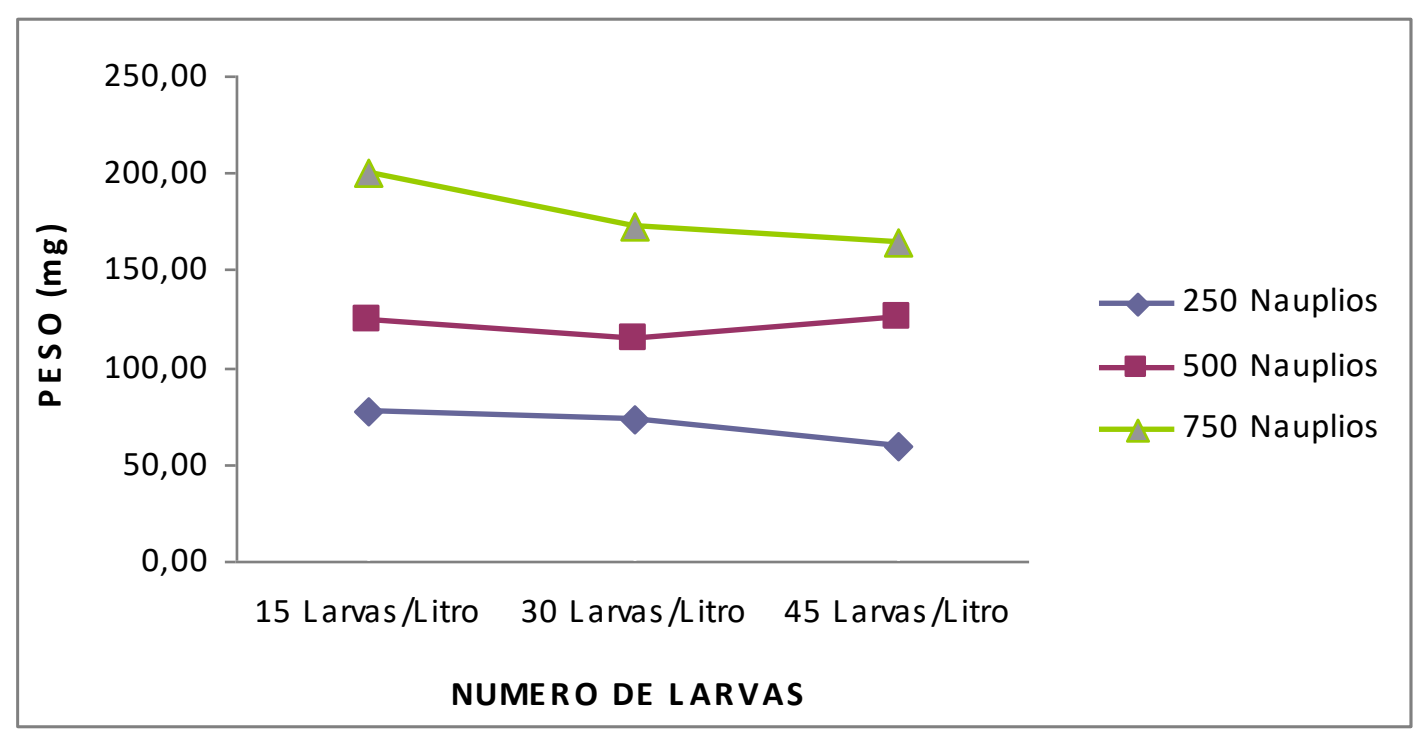

Figura 4. Ganancia de peso entre los factores densidad de siembra y concentración de Artemia

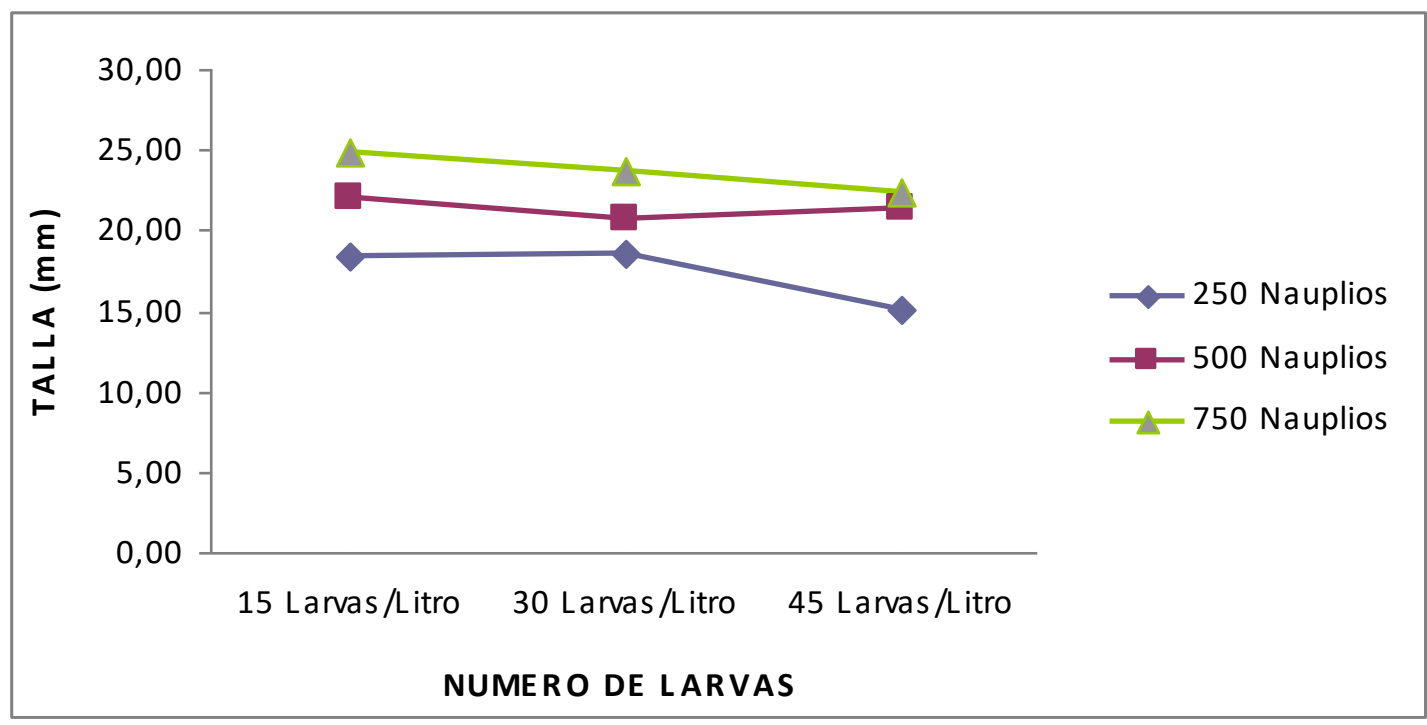

Figura 5. Ganancia longitud entre los factores densidad de siembra y concentración de Artemia

Ensayos sobre densidades de siembra en Perca fluvitilis, concluyeron que a densidades bajas, estos organismos desarrollan comportamientos territoriales, contrario a lo que ocurre en densidades altas donde el número de organismos caníbales es proporcionalmente bajo, explicado por la reducción de espacios que impiden el desarrollo de comportamientos territoriales (Kestemont et al., 2003); sin embargo, esto puede ser afectado cuando el alimento no es 
distribuido de forma homogénea, restringiendo el acceso de algunas larvas al recurso alimenticio (Baras y Jobling, 2002).

Según Qin y Fast, (1996) la conducta caníbal es inevitable en las especies carnívoras durante sus primeros estadios de vida, pero puede ser altamente reducida en juveniles, seleccionándolos por tallas y suministrándoles alimento ad libitum, pero evitando el suministro excesivo y asegurando una buena calidad de agua. En la experiencia con L. marmoratum, la reducción en la oferta de alimento a densidades altas, podría no afectar directamente el aumento en las conductas caníbales, pero si influir directamente sobre la ganancia de peso y talla; por tanto, la baja ganancia de peso y talla observada con bajas concentraciones de Artemia salina (250 nauplios.Larva-1), podría atribuirse a una alimentación insuficiente.

Los resultados obtenidos en el bagre sudamericano (Rhamdia quelen) indican que es posible reemplazar gradualmente a los nauplios de Artemia durante la alimentación de las larvas de bagre sudamericano sin afectar significativamente su crecimiento y sobrevivencia. Probablemente, la digestibilidad del alimento seco mejore gracias al aporte de algunos factores incluidos en el alimento vivo (ácidos grasos esenciales, neuropéptidos, amino ácidos libres), contribuyendo a una mejor asimilación de la ración balanceada, traduciéndose en buen crecimiento larval, sobrevivencia alta y disminución de costos y mano de obra que demanda el proceso de incubación de nauplios de Artemia. Finalmente, cabe mencionar que si se desea prolongar el período de alimentación de los peces en condiciones de laboratorio, la utilización de co-alimentación durante los primeros días seguramente redundará en mayores beneficios, ya que una vez que las larvas alimentadas con nauplios de Artemia alcanzan cierto tamaño, el gasto energético requerido para la captura de los mismos, supera al aporte que se obtiene de su digestión, deteniéndose por completo el crecimiento, lo que no ocurre si las larvas se alimentan con una ración balanceada de buena calidad (Hernández et al., 2005). En el trabajo experimental se observó que, en los días posteriores al día octavo, los animales consumían cerca de la totalidad de la ración en el menor tiempo, se infiere que sus requerimientos nutricionales no se satisfacían en este punto, se 
mejoraría haciendo un cambio paulatino a dietas balanceadas para satisfacer completamente sus requerimientos mejorando así, la ganancia de peso y talla a partir del día 8.

Los costos de producción utilizando Artemia como fuente de alimento en larvicultura son altos, siendo necesario estudiar alternativas como el uso de especies de zooplancton natural (Díaz et al., 2009) o dietas secas que proporcionen o tengan un comportamiento alimenticio similar al del alimento vivo; sin embargo, algunos trabajos reportan que en especies de hábitos piscívoros (depredación interespecífica), esta alternativa pasa a ser un recurso limitante luego de la primera semana de suministro, generando un crecimiento más heterogéneo debido a la baja digestibilidad y calidad nutricional de este tipo de alimento (Prieto y Atencio, 2008, Nuñez et al., 2008) quedando muchos individuos en desventaja con relación a aquellos que expresan su condición caníbal (Baras y Jobling, 2002).

\section{CONCLUSIONES}

Los resultados obtenidos en este experimento muestran que esta especie de silúridos tienen una época marcada de canibalismo entre el día 0 y el día 8 el cual puede reducirse brindando las condiciones necesarias para su crecimiento, sus requerimientos en esta etapa son una limitante ya que esta especie en sus primeros días son de hábitos cazadores el cual alimentos inertes no servirían para su alimentación.

El requerimiento de alimento vivo es una de las mayores limitantes en esta especie ya que los costos de este alimento son muy altos, además que en lo observado del ensayo se podría decir que no llenaba los requerimientos de la especie, debido a que se presentaba mucho canibalismo.

Este ensayo brindó información adicional del comportamiento de esta especie en un sistema. Las densidades manejadas respondieron directamente al tipo y cantidad de alimento utilizado dando como resultado que las densidades mas altas con una disponibilidad de alimento adecuada nos darían una mayor sobrevivencia, aunque su ganancia de peso y talla seria relativamente lenta, sin 
embargo, en estudios de otras especies esta ganancia de peso y talla esta en los niveles normales para especies de hábitos carnívoros.

Para la cadena comercial de silúridos de la región, estos resultados obtenidos favorecen en el mejoramiento del manejo tecnológico de esta especie, que sin duda representan una importante fuente de producción y comercialización.

\section{BIBLIOGRAFÍA}

1. Baras E.; Jobling M. Dynamics of intracohort cannibalism in cultured fish. Aquacult. Res. 33: 461 - 479. 2002.

2. Boiani L.; Bessonart M.; Berois N.; Salhi M. Desarrollo morfológico e histología del digestivo de larvas de bagre negro, Rhamdia quelen (pieces, pimelodidae). Bol. Soc. Zool. Uruguay, 2a época, 14: 17- 28. 2003.

3. Díaz O. J.; Cruz C. N.; Marciales C, L.; Medina R. V.; Cruz C. P. Efectos de la densidad de siembra y disponibilidad de alimento sobre el desarrollo y sobrevivencia de larvas de Pseudoplatystoma fasciatum, Revista Orinoquia, 13 (1): 21-30. 2009.

4. Gallo G. M.; García U. G. Crecimiento de crías de Oreochromis niloticus y Oreochromis mossambicus cultivadas en un sistema de recirculación y alimentadas con un suplemento de quistes de Artemia en la dieta comercial. Universidad de Colima. pp. 71-82. 2007.

5. Haylor G. S. Controlled hatchery production of Clarias gariepinus (Burchell, 1822): growth and survival of fry at night stocking density. Aquacult. Fisch. Manag.22: 405 - 422. 1991.

6. Hecht T.; Appelbaum S. Observations on intraspecific aggression and coeval sibling cannibalism by larva and juvenile Clarias gariepinus (Clariidae: Pisces) under controled conditions. Journal of Zoology, 214: 2144. 1988.

7. Hernández, D. R.; Flores, Q. C.; Domitrovic, H. A.; Bechara, J.; Sánchez, S. Evaluación de diferentes dietas en los primeros estadios del desarrollo del bagre sudamericano (Rhamdia quelen). Instituto de Ictiología del Nordeste, Facultad de Ciencias Veterinarias, UNNE; Resumen: V-026. 2005.

8. Jomori, R. K. Estudo sobre a alimentação de larvas de pacu, Piaractus mesopotamicus (Holemberg, 1887), com náuplios de artemia e a sua substituição por dieta artificial. 1999. 70 f. (Trabalho de graduação) Faculdade de Ciências Agrárias e Veterinárias, Universidade Estadual Paulista, Jaboticabal, 1999.

9. Katavic, I.; Judgujakovic, J.; Glamuzina, B. Cannibalism as a factor affecting the survival of intensively cultured sea bass (Dicentrarcus labrax) fingerlings. Aquaculture, 77: 135 - 143. 1989.

10.Kennedy R.; Zaniboni, E. Larvicultura do Mandi-amarelo Pimelodus maculates Lacépéde, 1803 (Siluriformes:Pimelodidae) em diferentes densidades de estocagem nos primeros días de Vida. R. Bras. Zootec. 31 (2): 560-565. 2002.

11. Kestemont, P.; Jourdan, S.; Houbart, C. C.; Paspatis, M.; Fontaine, P.; Cuvier, A.; Kentouri, M.; Baras, E. Size heterogeneity, cannibalism and 
competition in cultured predatory fish larvae: biotic and abiotic influences. Aquaculture, 227: 333-356. 2003.

12. Kestmont, P.; Stalmans, J. M. Initial feeding of European minnow larvae Phoxinus phoxinus L. 1. Influence of diet and feeding level. Aquaculture, 104: 327-340. 1992.

13. Khan M. S. Effect of population density on the growth feed and protein conversion efficiency and biochemical composition of a tropical freshwater catfish, Mystus nemurus (Curvier y Valenciennes). Aquacult. Fisch. Manag. 25: 753-760. 1994.

14. Kim L.; Lee S. M. Effects of the dietary protein and lipid levels on growth and body composition of bagrid catfish, Pseudobagrus fulvidraco. Aquaculture, 243: 323-329. 2005.

15. Lopes, J. M.; Silva, L.; Baldisserotto, B. Survival and growth of silver catfish larvae exposed to different water pH. Aquacult. Int. 9: 73-80. 2001.

16. Luchini, R.; Salas T. Primer alevinaje de bagre sudamericano Rhamdia sapo (Val.) Eig. en condiciones controladas. Rev. Asoc. Cienc. Nat Litoral, 16 (2): 137-147. 1985.

17. Nuñez, J.; Dugué R.; Corcuy A. N.; Duponchelle, F.; Renno, J. F.; Raynaud, T.; Hubert, N.; Legendre M. Induced breeding and larval rearing of Surubí, Pseudoplatystoma fasciatum (Linnaeus, 1766), from the Bolivian Amazon. Aquacult. Res. 39: 764-776. 2008.

18. Petrere, M.; Borges, R.; Agudelo E.; Corrales, B. Review of the large catfish fisheries in the upper Amazon and stock depletion of piraiba (Brachyplatystoma filamentosum Lichtenstein). Reviews in fish Biology and Fisheries, 14: 403-414. 2004.

19. Prieto, M.; Atencio, V. Zooplancton en la larvicultura de Peces Neotropicales. MVZ Córdoba,13 (2): 1415-1425. 2008.

20. Qin J.; Fast, A. W. Size and feed dependent cannibalism with juvenile snakehead Chana striatus. Aquaculture, 144: 313-320. 1996.

21. Segura, L.; Hayashi, C.; De Souza, S.; Soares, C. Canibalismo entre larvas de pintado, Pseudoplatystoma corruscans, cultivadas sob diferentes densidades de estocagem. Acta Scientiarum. Biological Sciences, 26 (3): 299-302. 2004. 